\title{
Altered secretome of Burkholderia pseudomallei induced by salt stress
}

\author{
Pornpan Pumirat ${ }^{\mathrm{a}}$, Putita Saetun ${ }^{\mathrm{b}}$, Supachok Sinchaikul ${ }^{\mathrm{c}}$, Shui-Tein Chen ${ }^{\mathrm{c}, \mathrm{d}}$, \\ Sunee Korbsrisate ${ }^{\mathrm{a}, *}$, Visith Thongboonkerd ${ }^{\mathrm{b}, *}$ \\ a Department of Immunology, Faculty of Medicine Siriraj Hospital, Mahidol University, 11th Floor Adulyadej Vikrom Building, 2 Prannok Road, Siriraj Hospital, Bangkoknoi, \\ Bangkok 10700, Thailand \\ ${ }^{\mathrm{b}}$ Medical Proteomics Unit, Office for Research and Development, Faculty of Medicine Siriraj Hospital, Mahidol University, Bangkok, Thailand \\ ${ }^{\mathrm{c}}$ Institute of Biological Chemistry and Genomic Research Center, Academia Sinica, Taipei, Taiwan \\ ${ }^{\mathrm{d}}$ Institute of Biochemical Sciences, College of Life Science, National Taiwan University, Taipei, Taiwan
}

\section{A R T I C L E I N F O}

\section{Article history:}

Received 9 September 2008

Received in revised form 2 December 2008

Accepted 29 January 2009

Available online 11 February 2009

\section{Keywords:}

Adaptive response

Burkholderia pseudomallei

Melioidosis

Proteome

Proteomics

Salt stress

Secretome

\begin{abstract}
A B S T R A C T
Burkholderia pseudomallei is a saprophyte found in soil and water. It is a difficult microorganism to kill and can survive in these environments for many years. Mechanisms for its adaptive response to environmental changes remain largely unknown. We performed a proteomics study to examine alterations in secreted proteins (secretome) under a salt stress (with $150 \mathrm{mM} \mathrm{NaCl}$ ) compared to the normal cultured condition in LB broth. The culture supernatants were filtrated and precipitated with 50\% ethanol. The isolated proteins were recovered, separated with 2-D PAGE, and visualized with SYPRO Ruby stain ( $n=5$ gels for each group). Differentially expressed protein spots were identified by Q-TOF MS and/or MS/MS analyses. A total of 42 protein spots representing 37 unique proteins were identified as the altered proteins during the salt stress, including metabolic enzymes, transcription/translation regulators, potential virulence factors, chaperones, phage capsid proteins, drug resistance protein, solute transport regulator, and hypothetical proteins. The presence of secreted GroEL only after $\mathrm{NaCl}$ exposure was confirmed by Western blot analysis. The increased level (19-fold) of a beta-lactamase-like protein suggested that the $\mathrm{NaCl}$-exposed bacterium might resist to beta-lactam antibiotics. Functional analysis revealed that the $\mathrm{NaCl}$-exposed bacterium had significantly greater survival rate after a treatment with ceftazidime. Our study provided the first dataset of the secretome of B. pseudomallei and its alterations, which may lead to novel insights into adaptive response of $B$. pseudomallei during the salt stress.
\end{abstract}

(c) 2009 Elsevier B.V. All rights reserved.

\section{Introduction}

Burkholderia pseudomallei is a Gram-negative saprophytic bacterium that causes melioidosis, which remains an important and common tropical disease, particularly in southeast Asia and northern Australia [1]. Melioidosis is most prevalent during rainy season in endemic areas $[2,3]$ and mainly affects individuals who have a direct contact with wet soil (especially those with an underlying disease, which predisposes to infection, such as diabetes, chronic renal failure, chronic lung disease, etc.) $[4,5]$. Clinical features of melioidosis vary largely and the disease course ranges from acute to subacute and chronic infection [1,5]. In the northeastern part of Thailand, the incidence of melioidosis is approximately 3.6-5.5 cases per 100,000 annually, with a high mortality rate for primary disease (up to $50 \%$ in adults and $35 \%$ in

\footnotetext{
* Corresponding authors. V. Thongboonkerd is to be contacted at Medical Proteomics Unit, Office for Research and Development, 12th Floor Adulyadej Vikrom Building, 2 Prannok Road, Siriraj Hospital, Bangkoknoi, Bangkok 10700, Thailand. Tel./fax: +66 2 4184793. S. Korbsrisate, Tel.: +66 2418 0569; fax: +66 24181636.

E-mail addresses: grsks@mahidol.ac.th (S. Korbsrisate), thongboonkerd@dr.com, vthongbo@yahoo.com (V. Thongboonkerd).
}

children) [1,4]. Unfortunately, there is no effective vaccine available for the prevention of melioidosis [6].

Melioidosis research during the past few decades has thus focused on exploring pathogenic and molecular mechanisms of this bacterial infection [7]. In addition, better understanding of the bacterial survival and virulence is also crucial for successful prevention and/or treatment of this infectious disease. Regarding its survival, $B$. pseudomallei, as a saprophyte, is a difficult microorganism to kill and can survive in soil and water for many years [4]. Therefore, it must have adaptive mechanisms to survive in these environments even with various stresses due to environmental changes (e.g., alterations in salt contents, osmolarity, $\mathrm{pH}$, etc.). However, these mechanisms of its adaptive response to environmental changes remain largely unknown.

The present study was therefore conducted to explore adaptive response in B. pseudomallei during a salt stress. After the stationary phase was reached, the bacteria were grown further with or without an addition of $\mathrm{NaCl}$ (at a final concentration of $150 \mathrm{mM}$ ) to the fresh culture medium. The secreted proteins were then isolated and analyzed by a gel-based proteomics method. Using this approach, a number of secreted proteins with altered levels were identified. Potential roles for the altered secretome in adaptive response during the salt stress are discussed. 


\section{Materials and methods}

\subsection{Bacterial culture and sample preparation}

B. pseudomallei (10276 strain) was maintained in Luria-Bertani (LB) broth at $37^{\circ} \mathrm{C}$. The growth phase was determined by measuring optical density (absorbance) at a wavelength of $600 \mathrm{~nm}$ for every $2 \mathrm{~h}$ using a UV-visible spectroscopy. After the stationary phase was reached (approximately 18-24 h), the bacteria were subcultured (with 1/10 dilution) in other sets of the fresh media and divided into two groups; with and without an addition of $\mathrm{NaCl}$ (to make a final concentration of $150 \mathrm{mM}$ ) ( $n=5$ replicates for each group). After $7 \mathrm{~h}$ of the growth in these two differential media, the bacteria were removed and the supernatants were saved by a centrifugation at $8000 \mathrm{~g}$ for $15 \mathrm{~min}$. The supernatants were then filtrated using MillexGP filter (Millipore; Bedford, MA) with a $0.22-\mu \mathrm{m}$ pore size. Secreted proteins in culture supernatants were precipitated using $50 \%$ ethanol. After another centrifugation at $13,000 \mathrm{~g}$ for $10 \mathrm{~min}$, the protein pellets were saved and the supernatants were discarded. The proteins in the pellets were then resuspended with a lysis buffer containing $7 \mathrm{M}$ urea, $2 \mathrm{M}$ thiourea, 4\% 3-[(3-cholamidopropyl) dimethyl-ammonio]-1propanesulfonate (CHAPS), $120 \mathrm{mM}$ dithiothreitol (DTT), 2\% ampho-

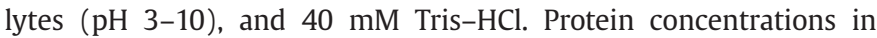
individual samples were then measured using the Bradford method.

\subsection{Two-dimensional polyacrylamide gel electrophoresis (2-D PAGE)}

A total of 10 gels derived from 10 individual culture flasks (5 from each group) were analyzed in the present study. Totally $100 \mu \mathrm{g}$ proteins derived from each culture flask were premixed with a rehydration buffer (7 M urea, 2 M thiourea, 2\% CHAPS, 120 mM DTT, 40 mM Tris-base, 2\% (v/ v) ampholytes ( $\mathrm{pH} \mathrm{3-10)} \mathrm{and} \mathrm{bromophenol} \mathrm{blue)} \mathrm{to} \mathrm{make} \mathrm{a} \mathrm{final}$ volume of $150 \mu \mathrm{l}$ per sample. The protein mixture was then rehydrated on immobilized pH gradient (IPG) strip, linear pH 3-10, 7-cm-long (GE Healthcare; Uppsala, Sweden) at room temperature for $16 \mathrm{~h}$. Subsequently, the first dimensional separation or isoelectric focusing (IEF) was performed using Ettan IPGphor II IEF System (GE Healthcare) with a step-and-hold mode until a total of $9083 \mathrm{Vh}$ was achieved. The IPG strip was then equilibrated with equilibration Buffer I ( $6 \mathrm{M}$ urea, $130 \mathrm{mM} \mathrm{DTT}$, 112 mM Tris-base, 4\% SDS, 30\% glycerol and 0.002\% bromophenol blue) for $15 \mathrm{~min}$, followed by another equilibration step in Buffer II ( $6 \mathrm{M}$ urea, $135 \mathrm{mM}$ iodoacetamide, $112 \mathrm{mM}$ Tris-base, 4\% SDS, 30\% glycerol and $0.002 \%$ bromophenol blue) for $15 \mathrm{~min}$. Thereafter, the strip was placed onto a $12 \%$ polyacrylamide slab gel $(8 \times 9.5 \mathrm{~cm})$, and the second dimensional separation was performed in SE260 mini-Vertical Electrophoresis Unit (GE Healthcare) at $150 \mathrm{~V}$ for approximately $2 \mathrm{~h}$. Separated proteins were then visualized using SYPRO Ruby fluorescence dye (Invitrogen - Molecular Probes; Eugene, OR), and 2-D gel images were obtained by Typhoon 9200 laser scanner (GE Healthcare).

\subsection{Matching and analysis of protein spots}

Matching and analysis of protein spots were performed using Image Master 2D Platinum software (GE Healthcare). Parameters used for spot detection were (i) minimal area $=10$ pixels; (ii) smooth factor $=2.0$; and (iii) saliency $=2.0$. A reference gel was created from an artificial gel combining all of the spots presenting in different gels into one image. The reference gel was then used for determination of existence and difference of protein expression among gels. Intensity volumes of individual spots were obtained and subjected to statistical analysis. Differentially expressed protein spots were subjected to ingel tryptic digestion and identification by mass spectrometry.

\subsection{Statistical analysis}

To define differentially expressed protein spots, unpaired Student's t-test was performed to compare intensity levels of corresponding spots between control and $\mathrm{NaCl}$ groups using SPSS software package for Windows (SPSS; Chicago, IL). The criteria for defining spots with significant differences (either increase or decrease) included (i) $p$-values $<0.05$, and (ii) the differentially expressed spots must be consistently present or absent in all five gels of each group.

\subsection{In-gel tryptic digestion}

The differentially expressed protein spots were excised from 2-D gels, washed twice with $200 \mu \mathrm{l}$ of $50 \%$ acetonitrile $(\mathrm{ACN}) / 25 \mathrm{mM}$ $\mathrm{NH}_{4} \mathrm{HCO}_{3}$ buffer ( $\mathrm{pH} \mathrm{8.0}$ ) at room temperature for $15 \mathrm{~min}$, and then washed once with $200 \mu \mathrm{l}$ of $100 \%$ ACN. After washing, the solvent was removed and the gel pieces were dried by a SpeedVac concentrator

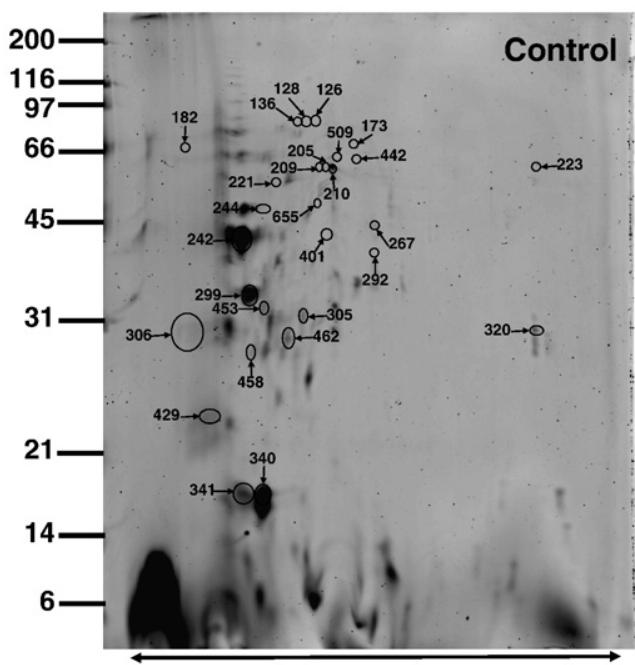

Linear $\mathrm{pH}$ gradient of 3-10

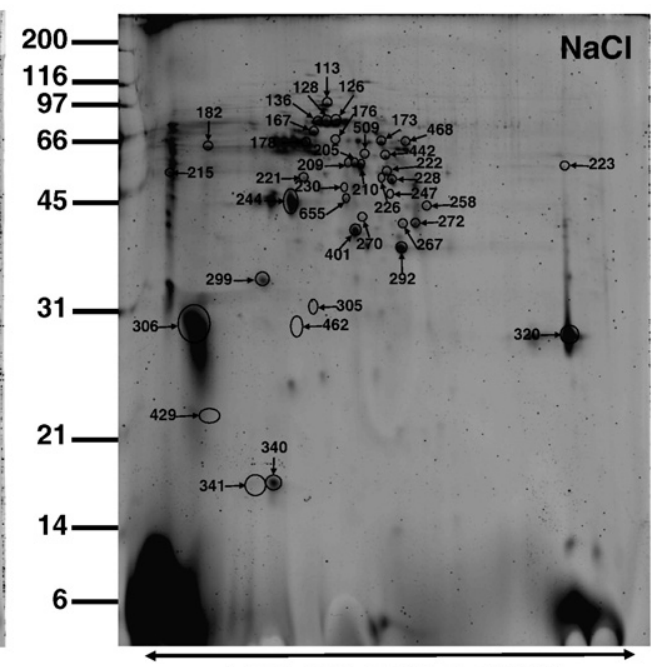

Linear $\mathrm{pH}$ gradient of $3-10$

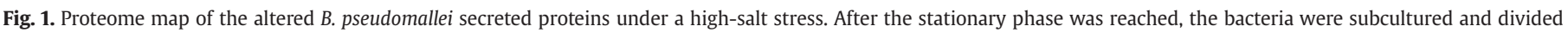

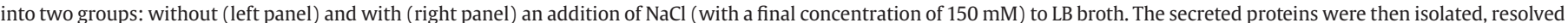

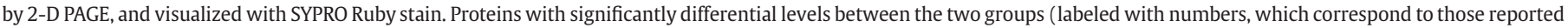

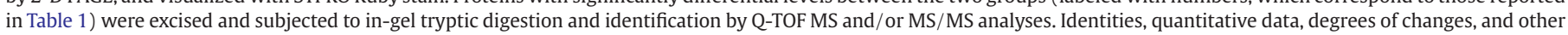
details of all these altered proteins are summarized in Table 1. 
Table 1

Altered B. pseudomallei secreted proteins induced by salt stress

\begin{tabular}{|c|c|c|c|c|c|c|c|c|c|c|c|c|}
\hline \multirow[t]{2}{*}{ Protein name } & \multirow{2}{*}{$\begin{array}{l}\text { Spot } \\
\text { no. }\end{array}$} & \multirow[t]{2}{*}{ NCBI ID } & \multirow{2}{*}{$\begin{array}{l}\text { Identified } \\
\text { by }\end{array}$} & \multirow{2}{*}{$\begin{array}{l}\text { Identification } \\
\text { scores } \\
\text { (MS, } \\
\text { MS/MS) }\end{array}$} & \multirow{2}{*}{$\begin{array}{l}\% \mathrm{Cov} \\
\text { (MS, } \\
\mathrm{MS} / \mathrm{MS})\end{array}$} & \multirow{2}{*}{$\begin{array}{l}\text { No. of } \\
\text { matched } \\
\text { peptides } \\
\text { (MS, } \\
\text { MS/MS) }\end{array}$} & \multirow[t]{2}{*}{$\mathrm{p} I$} & \multirow{2}{*}{$\begin{array}{l}\text { MW } \\
(\mathrm{kDa})\end{array}$} & \multicolumn{2}{|c|}{ Intensity (mean \pm SEM) } & \multirow{2}{*}{$\begin{array}{l}\text { Ratio } \\
\text { ( } \mathrm{NaCl} / \\
\text { control) }\end{array}$} & \multirow{2}{*}{$\begin{array}{l}p \\
\text { values }\end{array}$} \\
\hline & & & & & & & & & Control & $\mathrm{NaCl}$ & & \\
\hline \multicolumn{13}{|l|}{ Metabolic enzymes } \\
\hline $\begin{array}{l}\text { 3-oxoacyl-(acyl-carrier-protein) } \\
\text { synthase II }\end{array}$ & 655 & gi|86151671 & MS & 81, NA & 35, NA & 9, NA & 5.65 & 43.10 & $0.0128 \pm 0.0079$ & $0.2455 \pm 0.0558$ & 19.15 & 0.003 \\
\hline Acetyl-CoA acetyltransferase & 258 & gi|53719169 & MS & 95, NA & 39, NA & $12, \mathrm{NA}$ & 6.62 & 40.75 & $0.0000 \pm 0.0000$ & $0.1077 \pm 0.0445$ & $\mathrm{DIV} / 0$ & 0.042 \\
\hline $\begin{array}{l}\text { AMP-dependent synthetase } \\
\text { and ligase }\end{array}$ & 509 & gi|170744090 & MS & 83, NA & 34, NA & 10, NA & 6.06 & 54.33 & $0.0059 \pm 0.0059$ & $0.1612 \pm 0.0594$ & 27.41 & 0.032 \\
\hline $\begin{array}{l}\text { Arylsulfatase regulator } \\
\text { (Fe-S oxidoreductase) }\end{array}$ & 176 & gi|90408763 & MS & 77, NA & $28, \mathrm{NA}$ & $18, \mathrm{NA}$ & 7.16 & 51.35 & $0.0000 \pm 0.0000$ & $0.2603 \pm 0.0823$ & $\mathrm{DIV} / 0$ & 0.013 \\
\hline $\begin{array}{l}\text { ATP phosphoribosyl transferase } \\
\text { regulatory subunit }\end{array}$ & 299 & gi|110835059 & MS & 77, NA & $24, \mathrm{NA}$ & 8, NA & 5.02 & 42.71 & $8.5468 \pm 2.9556$ & $1.0521 \pm 0.3639$ & 0.12 & 0.036 \\
\hline ATPase & 458 & gi|56551020 & MS & 82, NA & 29, NA & $11, \mathrm{NA}$ & 5.30 & 58.69 & $0.3773 \pm 0.1316$ & $0.0000 \pm 0.0000$ & 0.00 & 0.021 \\
\hline ATPase, AAA family protein & 267 & gi|157165173 & MS & 85 , NA & 31, NA & $10, \mathrm{NA}$ & 6.30 & 44.36 & $0.0096 \pm 0.0096$ & $0.2411 \pm 0.0723$ & 25.20 & 0.013 \\
\hline Dehydratase protein & 221 & gi|21243028 & MS & 90, NA & $26, \mathrm{NA}$ & $12, \mathrm{NA}$ & 8.89 & 70.63 & $2.1656 \pm 0.2239$ & $0.4120 \pm 0.1029$ & 0.19 & $<0.001$ \\
\hline $\begin{array}{l}\text { D-isomer specific 2-hydroxyacid } \\
\text { dehydrogenase, NAD-binding }\end{array}$ & 306 & $\begin{array}{l}\text { gi| } \\
148544838\end{array}$ & MS & 59, NA & 32, NA & 7, NA & 4.80 & 36.60 & $0.0000 \pm 0.0000$ & $16.0833 \pm 0.8781$ & $\mathrm{DIV} / 0$ & $<0.001$ \\
\hline Glycosidase & 228 & gi|27367964 & MS & 70, NA & 27, NA & $10, \mathrm{NA}$ & 5.72 & 52.57 & $0.0000 \pm 0.0000$ & $0.3829 \pm 0.0604$ & $\mathrm{DIV} / 0$ & $<0.001$ \\
\hline $\begin{array}{l}\text { Isopentenyl-diphosphate } \\
\text { delta-isomerase }\end{array}$ & 341 & $\begin{array}{l}\text { gil } \\
149202049\end{array}$ & MS & 71, NA & $56, \mathrm{NA}$ & $8, N A$ & 6.54 & 19.49 & $2.8479 \pm 1.0513$ & $0.0495 \pm 0.0495$ & 0.02 & 0.029 \\
\hline Putative hydroxylase & 429 & gi|103487394 & MS & 63, NA & $45, \mathrm{NA}$ & $6, N A$ & 6.23 & 24.40 & $2.2644 \pm 0.7858$ & $0.0791 \pm 0.0791$ & 0.03 & 0.024 \\
\hline Putative hydroxylase & 462 & gi|103487394 & MS & 73, NA & 45 , NA & $8, \mathrm{NA}$ & 6.23 & 24.40 & $0.2034 \pm 0.0523$ & $0.0063 \pm 0.0063$ & 0.03 & 0.006 \\
\hline Putative methyltransferase & 242 & gi|84388938 & MS & 84, NA & 35, NA & $10, N A$ & 6.01 & 43.91 & $10.1691 \pm 1.8233$ & $0.0000 \pm 0.0000$ & 0.00 & 0.003 \\
\hline $\begin{array}{l}\text { Putative UDP-galactose } \\
\text { 4-epimerase }\end{array}$ & 292 & gi|68553183 & MS & 77, NA & 30, NA & 8, NA & 6.47 & 37.05 & $0.1251 \pm 0.0656$ & $0.8530 \pm 0.1982$ & 6.82 & 0.008 \\
\hline $\begin{array}{l}\text { Pyruvate dehydrogenase } \\
\text { complex dihydrolipoamide } \\
\text { acetyltransferase }\end{array}$ & 244 & gi|157372244 & MS & 75, NA & 30, NA & $10, \mathrm{NA}$ & 5.18 & 65.72 & $0.5910 \pm 0.3598$ & $4.1944 \pm 0.9829$ & 7.10 & 0.009 \\
\hline $\begin{array}{l}\text { Response regulator receiver } \\
\text { modulated metal dependent } \\
\text { phosphohydrolase }\end{array}$ & 453 & gi|118743860 & MS & 78, NA & 36, NA & 9, NA & 4.95 & 41.49 & $0.4248 \pm 0.1363$ & $0.0000 \pm 0.0000$ & 0.00 & 0.014 \\
\hline $\begin{array}{l}\text { S-adenosyl-L-homocysteine } \\
\text { hydrolase }\end{array}$ & 205 & gi|53720900 & MS, MS/MS & 85,7 & 26,2 & 10,1 & 5.73 & 52.51 & $0.0106 \pm 0.0106$ & $0.2600 \pm 0.0728$ & 24.44 & 0.010 \\
\hline $\begin{array}{l}\text { S-adenosyl-L-homocysteine } \\
\text { hydrolase }\end{array}$ & 210 & gi|53720900 & MS & 64, NA & 28, NA & $10, \mathrm{NA}$ & 5.73 & 52.51 & $0.0921 \pm 0.0921$ & $0.7126 \pm 0.1624$ & 7.74 & 0.010 \\
\hline \multicolumn{13}{|c|}{ Transcription and translation regulators } \\
\hline ATP-dependent RNA helicase & 209 & gi|53713588 & MS & 61, NA & 31, NA & $10, \mathrm{NA}$ & 5.84 & 46.93 & $0.0315 \pm 0.0253$ & $0.1890 \pm 0.0443$ & 6.00 & 0.015 \\
\hline Elongation factor EF-2 & 113 & gi|53720824 & MS & 85, NA & 27, NA & 13 , NA & 5.33 & 77.74 & $0.0000 \pm 0.0000$ & $0.4641 \pm 0.1753$ & $\mathrm{DIV} / 0$ & 0.029 \\
\hline Glutaminyl-tRNA synthetase & 226 & gi|66044979 & MS & 76, NA & 25 , NA & $10, \mathrm{NA}$ & 5.73 & 65.24 & $0.0000 \pm 0.0000$ & $0.0555 \pm 0.0173$ & $\mathrm{DIV} / 0$ & 0.012 \\
\hline $\begin{array}{l}\text { Glutamyl-tRNA(Gln) } \\
\text { amidotransferase, B subunit }\end{array}$ & 222 & gi|83319771 & MS & 87, NA & 23, NA & $10, \mathrm{NA}$ & 6.45 & 54.97 & $0.0000 \pm 0.0000$ & $0.1819 \pm 0.0621$ & $\mathrm{DIV} / 0$ & 0.019 \\
\hline GTP-binding protein LepA & 215 & gi|24379799 & MS & 74, NA & 27, NA & $10, N A$ & 5.16 & 68.54 & $0.0000 \pm 0.0000$ & $0.1532 \pm 0.0430$ & $\mathrm{DIV} / 0$ & 0.007 \\
\hline Transcriptional regulator & 320 & gi|84357351 & MS & 55, NA & 25 , NA & 4, NA & 6.19 & 30.44 & $0.5721 \pm 0.4213$ & $7.4625 \pm 1.8963$ & 13.04 & 0.008 \\
\hline Transposase & 305 & gi|58701083 & MS & 88, NA & 43 , NA & $8, N A$ & 8.40 & 23.21 & $0.8037 \pm 0.1795$ & $0.0767 \pm 0.0400$ & 0.10 & 0.004 \\
\hline $\begin{array}{l}\text { tRNA } \\
\text { (uracil-5-)-methyltransferase } \\
\text { Gid }\end{array}$ & 182 & gi|86749845 & MS & 70, NA & 29, NA & $9, \mathrm{NA}$ & 6.68 & 51.43 & $0.0091 \pm 0.0091$ & $0.3487 \pm 0.0916$ & 38.36 & 0.006 \\
\hline tRNA nucleotidyl transferase & 247 & gi|134096102 & MS & $64, \mathrm{NA}$ & 27, NA & 8, NA & 6.41 & 46.43 & $0.0000 \pm 0.0000$ & $0.0249 \pm 0.0102$ & $\mathrm{DIV} / 0$ & 0.040 \\
\hline \multicolumn{13}{|l|}{ Potential virulence factors } \\
\hline Peptidase, M1 family & 126 & gi|134283293 & MS, MS/MS & 116,32 & 28,3 & 15,2 & 6.74 & 82.14 & $0.1035 \pm 0.0935$ & $1.1483 \pm 0.3801$ & 11.09 & 0.028 \\
\hline Peptidase, M1 family & 128 & gi|134283293 & MS, MS/MS & 99,23 & 21,1 & 14,1 & 6.74 & 82.14 & $0.1379 \pm 0.1086$ & $1.3742 \pm 0.4828$ & 9.97 & 0.037 \\
\hline Peptidase, M1 family & 136 & gi|134283293 & MS, MS/MS & 94,24 & 24,1 & 14,1 & 6.74 & 82.14 & $0.0116 \pm 0.0116$ & $1.6693 \pm 0.7036$ & 143.85 & 0.046 \\
\hline Peptidase, M1 family & 173 & gi|134283293 & MS & $84, \mathrm{NA}$ & 21, NA & $12, \mathrm{NA}$ & 6.74 & 82.14 & $0.0058 \pm 0.0058$ & $0.1253 \pm 0.0504$ & 21.79 & 0.046 \\
\hline \multicolumn{13}{|l|}{ Chaperones } \\
\hline GroEL & 178 & gi|28630950 & MS, MS/MS & 197,100 & 60,6 & 20,2 & 5.18 & 56.49 & $0.0000 \pm 0.0000$ & $1.2901 \pm 0.2463$ & $\mathrm{DIV} / 0$ & 0.012 \\
\hline $\begin{array}{l}\text { Heat shock protein } \\
\text { transcription repressor }\end{array}$ & 272 & gi|157693049 & MS & $62, N A$ & 18, NA & $5, N A$ & 5.96 & 38.90 & $0.0000 \pm 0.0000$ & $0.3754 \pm 0.1150$ & $\mathrm{DIV} / 0$ & 0.011 \\
\hline \multicolumn{13}{|l|}{ Phage capsid proteins } \\
\hline $\begin{array}{l}\text { Phage major capsid } \\
\text { protein precursor }\end{array}$ & 270 & gi|53717812 & MS, MS/MS & 85,35 & 33,3 & 11,1 & 6.78 & 38.18 & $0.0000 \pm 0.0000$ & $0.1176 \pm 0.0329$ & $\mathrm{DIV} / 0$ & 0.007 \\
\hline Major capsid protein precursor & 401 & gi|53722091 & MS, MS/MS & 141,54 & 37,9 & 15,2 & 6.78 & 38.22 & $0.0286 \pm 0.0286$ & $0.6991 \pm 0.1473$ & 24.42 & 0.002 \\
\hline \multicolumn{13}{|l|}{ Drug resistance protein } \\
\hline Beta-lactamase-like protein & 442 & gi|149914600 & MS & $76, \mathrm{NA}$ & 27, NA & 11, NA & 5.50 & 60.76 & $0.0076 \pm 0.0076$ & $0.1464 \pm 0.0689$ & 19.29 & 0.008 \\
\hline \multicolumn{13}{|c|}{ Solute transport regulatory protein } \\
\hline $\begin{array}{l}\text { Bacterial extracellular } \\
\text { solute-binding protein }\end{array}$ & 223 & gi|33599920 & MS & 59, NA & 24, NA & $10, \mathrm{NA}$ & 6.33 & 58.85 & $0.0112 \pm 0.0112$ & $0.0968 \pm 0.0288$ & 8.61 & 0.025 \\
\hline
\end{tabular}


Table 1 (continued)

\begin{tabular}{|c|c|c|c|c|c|c|c|c|c|c|c|c|}
\hline \multirow[t]{2}{*}{ Protein name } & \multirow{2}{*}{$\begin{array}{l}\text { Spot } \\
\text { no. }\end{array}$} & \multirow[t]{2}{*}{ NCBI ID } & \multirow{2}{*}{$\begin{array}{l}\text { Identified } \\
\text { by }\end{array}$} & \multirow{2}{*}{$\begin{array}{l}\text { Identification } \\
\text { scores } \\
\text { (MS, } \\
\text { MS/MS) }\end{array}$} & \multirow{2}{*}{$\begin{array}{l}\% \text { Cov } \\
\text { (MS, } \\
\text { MS/MS) }\end{array}$} & \multirow{2}{*}{$\begin{array}{l}\text { No. of } \\
\text { matched } \\
\text { peptides } \\
\text { (MS, } \\
\text { MS/MS) }\end{array}$} & \multirow[t]{2}{*}{$\mathrm{p} I$} & \multirow{2}{*}{$\begin{array}{l}\text { MW } \\
\text { (kDa) }\end{array}$} & \multicolumn{2}{|c|}{ Intensity (mean $\pm \mathrm{SEM}$ ) } & \multirow{2}{*}{$\begin{array}{l}\text { Ratio } \\
\text { (NaCl/ } \\
\text { control) }\end{array}$} & \multirow{2}{*}{$\begin{array}{l}p \\
\text { values }\end{array}$} \\
\hline & & & & & & & & & Control & $\mathrm{NaCl}$ & & \\
\hline \multicolumn{13}{|l|}{ Hypothetical protein } \\
\hline $\begin{array}{l}\text { Hypothetical protein } \\
\text { Bpse110_02001906 }\end{array}$ & 340 & gi|82536900 & MS & 72, NA & 48, NA & $7, \mathrm{NA}$ & 4.92 & 16.08 & $15.4537 \pm 3.8195$ & $2.5137 \pm 0.3937$ & 0.16 & 0.010 \\
\hline $\begin{array}{l}\text { Hypothetical protein } \\
\text { BURPSPAST_T0419 }\end{array}$ & 468 & gi|157934517 & MS & $86, \mathrm{NA}$ & 33, NA & $13, \mathrm{NA}$ & 6.39 & 56.53 & $0.0000 \pm 0.0000$ & $0.3126 \pm 0.1011$ & $\mathrm{DIV} / 0$ & 0.015 \\
\hline Hypothetical protein mlr0590 & 230 & gi|13470794 & MS & $58, \mathrm{NA}$ & 14 , NA & $6, \mathrm{NA}$ & 5.77 & 60.10 & $0.0000 \pm 0.0000$ & $0.0294 \pm 0.0127$ & $\mathrm{DIV} / 0$ & 0.049 \\
\hline Hypothetical protein X004849 & 167 & gi|116247043 & MS & 72, NA & 23, NA & 8, NA & 8.48 & 60.57 & $0.0000 \pm 0.0000$ & $0.6520 \pm 0.2517$ & $\mathrm{DIV} / 0$ & 0.032 \\
\hline
\end{tabular}

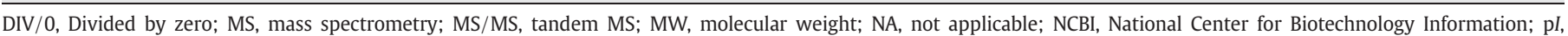
Isoelectric point.

(Savant; Holbrook, NY). The dried gel plugs were then rehydrated with $10 \mu \mathrm{l}$ of $1 \%(\mathrm{w} / \mathrm{v}$ ) trypsin (Promega; Madison, WI) in $25 \mathrm{mM}$ $\mathrm{NH}_{4} \mathrm{HCO}_{3}$. After rehydration, the gel pieces were crushed with siliconized blue stick and incubated at $37{ }^{\circ} \mathrm{C}$ for at least $16 \mathrm{~h}$. Peptides were subsequently extracted twice with $50 \mu \mathrm{l}$ of $50 \% \mathrm{ACN} / 5 \%$ trifluoroacetic acid (TFA); the extracted solutions were then combined

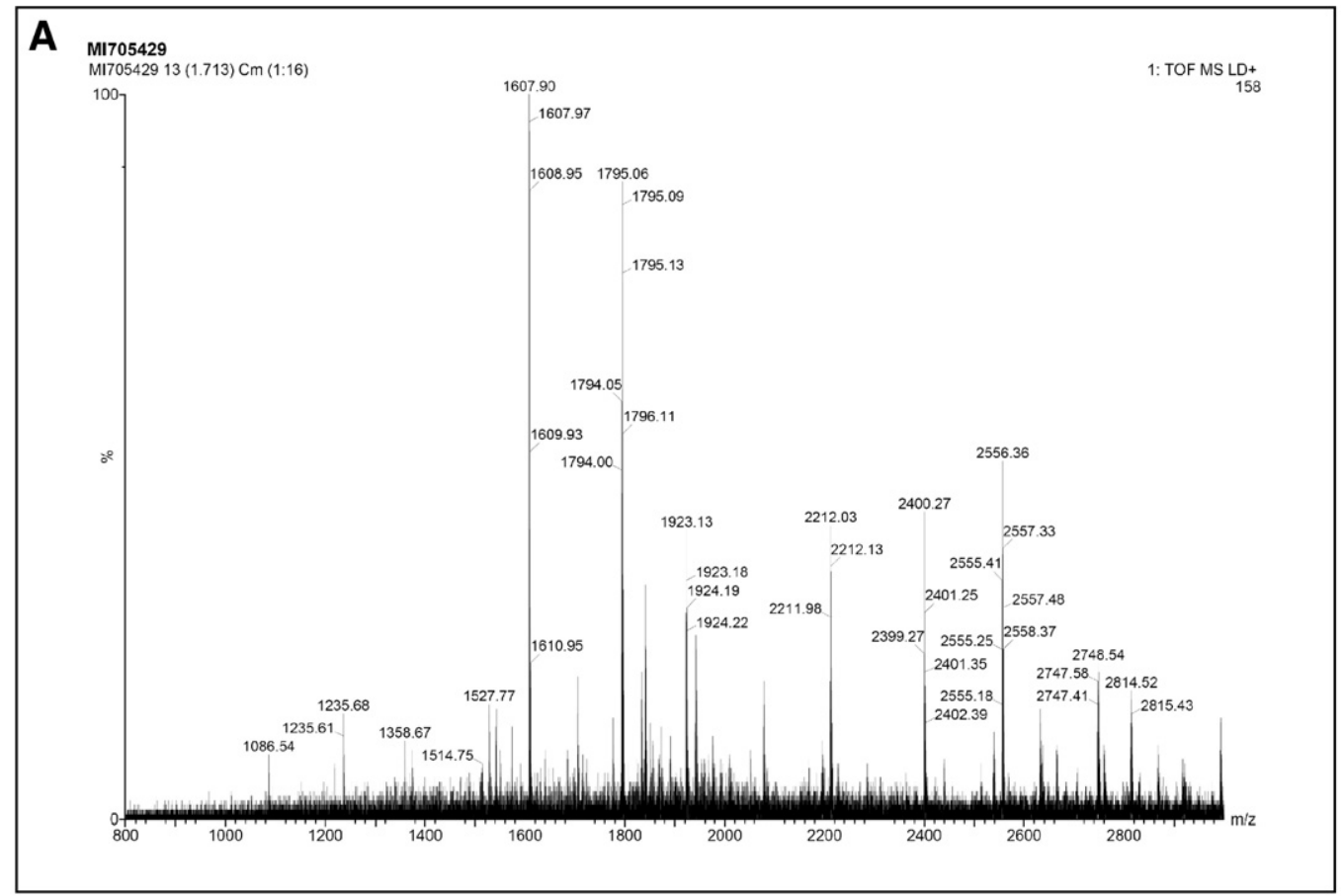

\begin{tabular}{|c|c|c|c|c|c|c|c|}
\hline Start & - End & Observed & $\operatorname{Mr}($ expt) & $\operatorname{Mr}(\mathrm{calc})$ & ppm & Miss & Sequence \\
\hline 2 & -13 & 1235.6333 & 1234.6260 & 1234.6306 & -4 & 1 & M. AAKDVVFGDSAR . A \\
\hline 14 & -28 & 1572.8392 & 1571.8319 & 1571.8705 & -25 & 1 & R. AKMVEGVNILANAVK. V* \\
\hline 16 & -34 & 1953.0045 & 1951.9972 & 1952.1129 & -59 & 1 & K. MVEGVNILANAVKVTLGPK. G \\
\hline 43 & -58 & 1549.7974 & 1548.7901 & 1548.8148 & -16 & 1 & R.SFGGPTVTKDGVSVAK.E \\
\hline 81 & -101 & 2078.0254 & 2077.0181 & 2077.0288 & -5 & 0 & K.TSDNAGDGTTTATVLAQSIVR. E \\
\hline 143 & -161 & 1889.9163 & 1888.9090 & 1888.9126 & -2 & 0 & K.EIAQVGAISANSDSSIGDR.I \\
\hline 172 & -197 & 2867.3647 & 2866.3574 & 2866.3542 & 1 & 1 & K. EGVITVEDGKSLADELDVVEGMQFDR . G* \\
\hline 182 & -197 & 1839.8452 & 1838.8379 & 1838.8356 & 1 & 0 & K.SLADELDVVEGMQFDR.G* \\
\hline 198 & -210 & 1527.7499 & 1526.7426 & 1526.7405 & 1 & 0 & R.GYLSPYFINNPDK.Q \\
\hline 227 & -242 & 1794.0376 & 1793.0303 & 1793.0411 & -6 & 1 & K.VSNIRDLLPVLEQVAK . A \\
\hline 243 & -268 & 2746.5140 & 2745.5067 & 2745.5388 & -12 & 0 & K. AGRPLLIIAEDVEGEALATLVVNNIR.G \\
\hline 286 & -311 & 2759.4595 & 2758.4522 & 2758.4674 & -5 & 1 & R. KAMLEDIAILTGGQVIAEETGLTLEK . A * \\
\hline 287 & -311 & 2631.3484 & 2630.3411 & 2630.3724 & -12 & 0 & K. AMLEDIAILTGGQVIAEETGLTLEK . A * \\
\hline 323 & -345 & 2399.2305 & 2398.2232 & 2398.2339 & -4 & 1 & R.IEVGKENTTIIDGAGEAVNIEAR.V \\
\hline 348 & -362 & 1824.8748 & 1823.8675 & 1823.8649 & 1 & 1 & K.QIRTQIEEATSDYDR.E \\
\hline 351 & -364 & 1684.7893 & 1683.7820 & 1683.7587 & 14 & 1 & R.TQIEEATSDYDREK.L \\
\hline 405 & -421 & 1607.9108 & 1606.9035 & 1606.9043 & -0 & 0 & R. AAVEEGIVPGGGVALIR. A \\
\hline 424 & -445 & 2225.0896 & 2224.0823 & 2224.2539 & -77 & 1 & R. TAIASLTGVNADQNAGIKIVLR. A \\
\hline 474 & -501 & 2992.3872 & 2991.3799 & 2991.3920 & -4 & 1 & K. GNYGYNAATGEYVDMVEAGVVDPTKVTR . T* \\
\hline 502 & -526 & 2438.3027 & 2437.2954 & 2437.3428 & -19 & 0 & R.TALQNAASVAGLLLTTDAAVAELPK .E \\
\hline
\end{tabular}

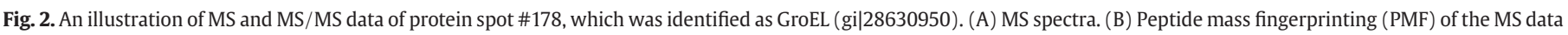

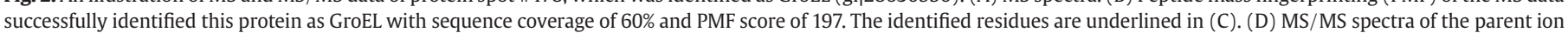

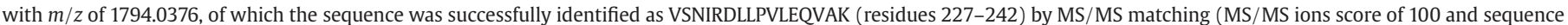
coverage of $6 \%$ ) as demonstrated in (E). * = Oxidation at methionine residue. 

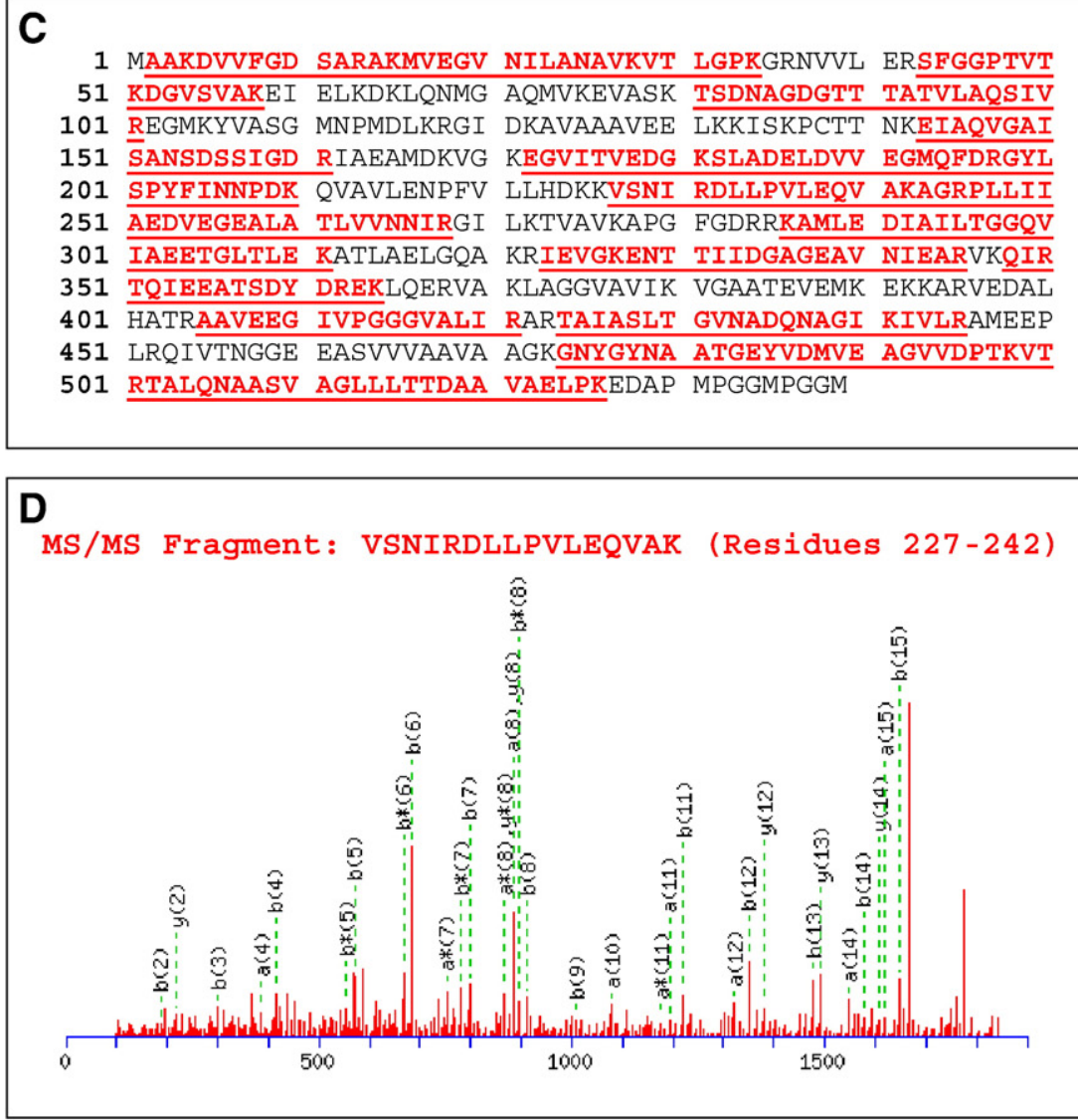

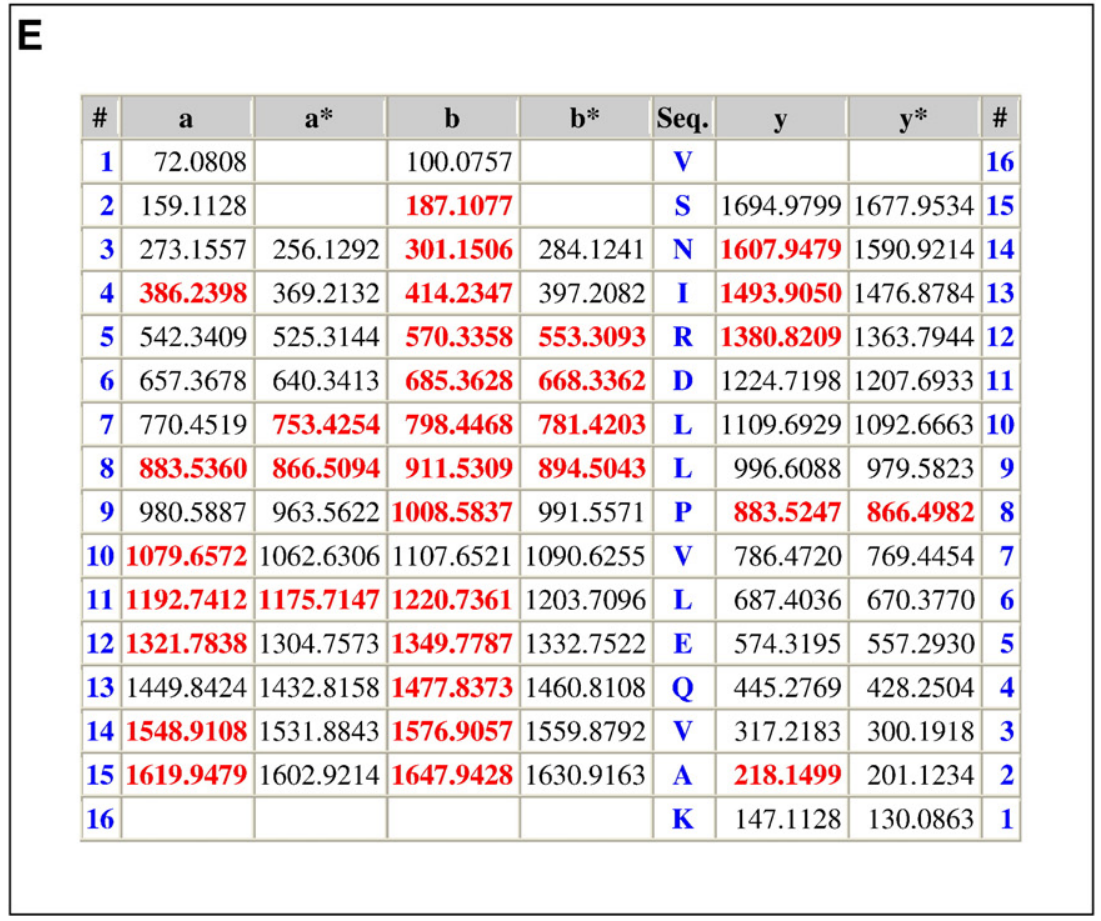

Fig. 2 (continued).

and dried with the SpeedVac concentrator. The peptide pellets were resuspended with $10 \mu \mathrm{l}$ of $0.1 \%$ TFA and purified using ZipTip C18 $_{18}$ (Millipore). The peptide solution was drawn up and down in the ZipTip $_{\mathrm{c} 18} 10$ times and then washed with $10 \mu \mathrm{l}$ of $0.1 \%$ formic acid by drawing up and expelling the washing solution three times. The peptides were finally eluted with $5 \mu$ of $75 \% \mathrm{ACN} / 0.1 \%$ formic acid.

\subsection{MS and/or MS/MS analyses}

The trypsinized samples were premixed 1:1 with the matrix solution containing $5 \mathrm{mg} / \mathrm{ml} \alpha$-cyano-4-hydroxycinnamic acid (CHCA) in 50\% ACN, $0.1 \%(\mathrm{v} / \mathrm{v})$ TFA and $2 \%(\mathrm{w} / \mathrm{v})$ ammonium citrate, and deposited onto the 96-well MALDI target plate. The samples were analyzed by Q- 
TOF Ultima ${ }^{\mathrm{TM}}$ mass spectrometer (Micromass; Manchester, UK), which was fully automated with predefined probe motion pattern and the peak intensity threshold for switching over from MS survey scanning to MS/MS, and from one MS/MS to another. Within each sample well, parent ions that met the predefined criteria (any peak within the $m / z$ 800-3000 range with intensity above 10 count \pm include/exclude list) were selected for CID MS/MS using argon as the collision gas and a mass dependent $\pm 5 \mathrm{~V}$ rolling collision energy until the end of the probe pattern was reached. The MS and MS/MS data were extracted and outputted as the searchable .txt and .pkl files, respectively, for independent searches using the MASCOT search engine (http://www. matrixscience.com), assuming that peptides were monoisotopic. Fixed modification was carbamidomethylation at cysteine residues, whereas variable modification was oxidation at methionine residues. Only one missed trypsin cleavage was allowed, and peptide mass tolerances of 100 and $50 \mathrm{ppm}$ were allowed for peptide mass fingerprinting and MS/MS ions search, respectively.

\subsection{Western blot analysis}

Totally $20 \mu$ g proteins extracted from each sample were resolved with SDS-PAGE at $150 \mathrm{~V}$ for approximately $2 \mathrm{~h}$ using SE260 miniVertical Electrophoresis Unit (GE Healthcare). After the completion of SDS-PAGE, proteins were transferred onto a nitrocellulose membrane and non-specific bindings were blocked with $5 \%$ milk in PBS for $1 \mathrm{~h}$. The membrane was then incubated with mouse monoclonal anti-GroEL (60 kDa chaperonin or HSP60) antibody (1:500 in 5\% milk/PBS) (Santa Cruz Biotechnology, Inc.; Santa Cruz, CA) at $4{ }^{\circ} \mathrm{C}$ overnight. After washing, the membrane was further incubated with rabbit anti-mouse IgG conjugated with horseradish peroxidase (1:1000 in 5\% milk/PBS) (Dako; Glostrup, Denmark) at room temperature for $1 \mathrm{~h}$. Reactive protein bands were then visualized with SuperSignal ${ }^{\circledR}$ West Pico chemiluminescence substrate (Pierce Biotechnology, Inc.; Rockford, IL).

\subsection{Evaluation of bacterial survival after treatment with a beta-lactam antibiotic}

Assay to evaluate beta-lactamase activity was performed by measuring bacterial survival after an exposure to ceftazidime, which is a beta-lactam antibiotic. B. pseudomallei 10276 strain was maintained in LB broth as aforementioned. After $7 \mathrm{~h}$ of culture in two differential media (with or without $\mathrm{NaCl}$ ), both groups were treated with ceftazidime (Millimed; Samut Prakarn, Thailand) at the final concentration of $1000 \mu \mathrm{g} / \mathrm{ml}$. Their viable colony forming units (CFU) were counted before and after 2-h treatment with ceftazidime. Percentage of bacterial survival was determined using the formula: \%Survival= $\left[\mathrm{CFU}_{\text {(after ceftazidime treatment) }} / \mathrm{CFU}_{\text {(before ceftazidime treatment) }}\right] \times 100 \%$.

\section{Results and discussion}

We evaluated changes in the secretome of $B$. pseudomallei under an exposure to the high-salt environment (by an addition of $\mathrm{NaCl}$ into the LB broth at a final concentration of $150 \mathrm{mM}$ ). To the best of our knowledge, this is the first study characterizing the secretome of B. pseudomallei. After $7 \mathrm{~h}$ of the exposure to salt stress, the 2-D proteome profile of the $\mathrm{NaCl}$-exposed bacterium was compared to that of the control ( $n=5$ replicates for each group). Using 2-D PAGE and SYPRO Ruby fluorescence stain, $93 \pm 21$ spots were visualized in the $\mathrm{NaCl}$-exposed group, whereas $148 \pm 14$ spots were detected in the controls. Image Master 2D Platinum software was employed to compare the protein spot pattern in all 2-D gels (5 gels in each group; totally 10 gels were analyzed). Quantitative intensity analysis and statistics revealed significant differences in spot intensity levels of totally 42 protein spots between the two groups (Fig. 1). All these significant differences had $p$ values $<0.05$, and were consistently present or absent in all five gels of each group.
Among these, 17 spots had increased levels $(\mathrm{NaCl} /$ control relative abundance ratios were ranged from 6.00 to 143.85 fold), 7 spots had decreased levels $(\mathrm{NaCl} /$ control relative abundance ratios were ranged from 0.02 to 0.19 fold), 3 spots were absent, and 15 spots were present only after an exposure to $\mathrm{NaCl}$ (Table 1 and Fig. 1 ). These data indicate the high degree of alterations in secretome of $B$. pseudomallei under a salt stress. These altered proteins were then successfully identified by Q-TOF MS and/or MS/MS analyses. Most of the altered proteins identified in this study were metabolic enzymes, transcription/ translation regulators, and potential virulence factors. Other altered proteins included chaperones, drug resistance protein, solute transport regulator, and hypothetical proteins. Fig. 2 illustrates MS and MS/MS analyses of spot \#178, which was identified as GroEL (gi|28630950). Fig. 2A illustrates MS spectra of this spot, whereas Fig. 2B and C demonstrates peptide mass fingerprinting (PMF) of this spot with a matching score of 197 and sequence coverage of 60\% (from totally 20 significantly matched peptides). Fig. 2D and E shows the MS/MS matching of GroEL with a MS/MS ion score of 100 and sequence coverage of $6 \%$ (from 2 significantly matched peptides). Fig. 3 shows Western blot data, which confirmed the presence of GroEL in the culture supernatant only after an exposure to $\mathrm{NaCl}$.

Recently, the complete genome sequence and annotation of B. pseudomallei has been made available [8,9], allowing the postgenomic characterizations of $B$. pseudomallei feasible and leading to a wider biological view of this microorganism. Based on the genomic annotation and translation, proteins that are associated with the survival of B. pseudomallei include those involving in secondary metabolism (i.e., antibiotic, surfactant, and siderophore biosynthetic pathways); drug resistance (i.e., beta-lactamases, multidrug efflux pumps, and aminoglycoside acetyltransferase); intracellular stress (i.e., superoxide and nitric oxide detoxification enzymes); and motility and chemotaxis (i.e., flagella proteins and chemotaxis-associated proteins) $[4,7,8]$. We identified a 19-fold increase of beta-lactamase-like protein during an exposure to the high-salt environment. The increase in secreted beta-

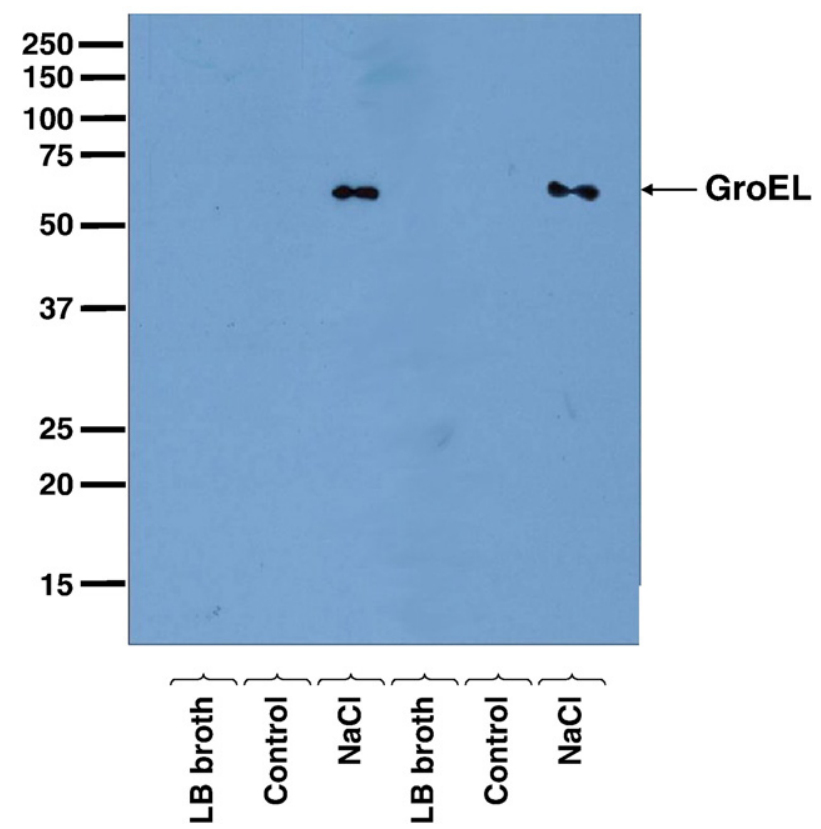

Fig. 3. Western blot analysis for GroEL. Totally $20 \mu \mathrm{g}$ proteins extracted from each sample were resolved by SDS-PAGE and transferred onto a nitrocellulose membrane. Basal LB broth (prior to bacterial culture) also served as another control. After blocking, the membrane was incubated with mouse monoclonal anti-GroEL (60 kDa chaperonin or HSP60) antibody (1:500 in 5\% milk in PBS) at $4{ }^{\circ} \mathrm{C}$ overnight and then with rabbit anti-mouse IgG conjugated with horseradish peroxidase (1:1000 in 5\% milk in PBS) at room temperature for $1 \mathrm{~h}$. Reactive protein bands were then visualized with SuperSignal ${ }^{\circledR}$ West Pico chemiluminescence substrate. 


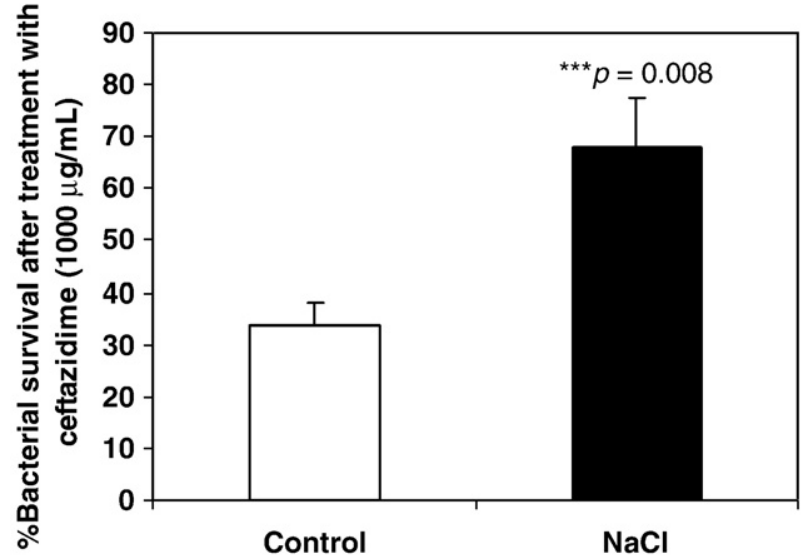

Fig. 4. Evaluation of bacterial survival after treatment with ceftazidime. B. pseudomallei 10276 strain was maintained in LB broth as detailed in "Materials and methods". After $7 \mathrm{~h}$ of culture in two differential media (with or without $\mathrm{NaCl}$ ), both groups were treated with $1000 \mu \mathrm{g} / \mathrm{ml}$ ceftazidime. Their viable colony forming units (CFU) were counted before and after 2-h treatment with ceftazidime. Percentage of bacterial survival was determined using the formula: \%Survival $=\left[\mathrm{CFU}_{(\text {after ceftazidime treatment })} /\right.$ $\left.\mathrm{CFU}_{\text {(before ceftazidime treatment) }}\right] \times 100 \%$.

lactamases or beta-lactamase-like proteins in culture supernatant might reflect the adaptive response of $B$. pseudomallei under the salt stress to resist to beta-lactam antibiotics $[10,11]$. We therefore performed a functional analysis to compare the bacterial survival after treatment with $1000 \mu \mathrm{g} / \mathrm{ml}$ ceftazidime, a beta-lactam antibiotic, in $\mathrm{NaCl}$-exposed bacterium versus control. Fig. 4 demonstrates that the $\mathrm{NaCl}$-exposed bacterium had significantly greater survival rate after ceftazidime treatment, indicating its resistance to ceftazidime (betalactam antibiotic) induced by the salt stress. Indeed, most of the identified proteins that were altered during an exposure to $\mathrm{NaCl}$ included metabolic enzymes and transcription/translation regulators, which are very important for bacterial growth and survival. Most of them were either increased or newly present after an exposure to $\mathrm{NaCl}$, implicating their important roles in adaptive response.

During the environmental stresses, several of partly folded cellular proteins tended to aggregate with others via their exposed hydrophobic regions [12]. To combat or prevent this stress-induced effect, the chaperonin GroEL (60 kDa chaperonin or HSP60) together with GroES form a folding cage to encapsulate the individual polypeptide chains to allow these polypeptide chains to fold until the hydrophobic regions are buried within the completely folded structure [12-14]. We identified an increased level of secreted GroEL during the salt stress. This increase might reflect adaptive response of $B$. pseudomallei to prevent protein aggregation induced by salt stress. While GroEL level was increased, we observed the increased level of a protein that prevents the transcription of heat shock proteins in our model. The mechanism underlying this disparate result remains unknown and should be further elucidated.

Apart from the proteins that are involved in the survival as aforementioned, proteins that are associated with the virulence of B. pseudomallei include type I, II, III and IV secretion systems; surface components (i.e., lipopolysaccharide, capsular polysaccharide and potential surface polysaccharide biosynthesis); exoproteins (i.e., phospholipase C, metalloproteases A, collagenase and other proteases); fimbriae/pili; and adhesins or adhesive molecules that modulate host-cell interaction [4,7,8]. We identified 4 forms of peptidase in M1 family, which is a membrane protease, with increased levels (ranged from 9.97 to 143.85 fold that is the maximal degree of changes identified in this study). These findings provided the first evidence demonstrating that salt stress can enhance the secretion of these potential virulence factors of $B$. pseudomallei.

In summary, we report herein the first dataset of the secretome of B. pseudomallei and its alterations during salt stress. These findings may provide some novel insights into the adaptive response of this microorganism to survive during the salt stress. Further characterizations and functional analyses of these altered proteins may lead to identification of new therapeutic targets or vaccine development for melioidosis.

\section{Acknowledgements}

We are grateful to Napat Songtawee, Theptida Semangoen, and Veerachat Muangsombut for their technical assistance. We also thank the Core Facilities for Proteomics and Structural Biology Research, Institute of Biological Chemistry, Academia Sinica, Taiwan. This study was supported by Siriraj Grant for Research and Development, Commission on Higher Education, and The Thailand Research Fund (Grant RMU 5080015) (to S. Korbsrisate and V. Thongboonkerd). P. Pumirat was supported by Siriraj Graduate Scholarship and by the Royal Golden Jubilee Ph.D. Program.

\section{References}

[1] N.J. White, Melioidosis, Lancet 361 (2003) 1715-1722.

[2] B.J. Currie, S.P. Jacups, Intensity of rainfall and severity of melioidosis, Australia, Emerg. Infect. Dis. 9 (2003) 1538-1542.

[3] B.J. Currie, S.P. Jacups, A.C. Cheng, D.A. Fisher, N.M. Anstey, S.E. Huffam, V.L. Krause, Melioidosis epidemiology and risk factors from a prospective whole-population study in northern Australia, Trop. Med. Int. Health 9 (2004) 1167-1174.

[4] A.C. Cheng, B.J. Currie, Melioidosis: epidemiology, pathophysiology, and management, Clin. Microbiol. Rev. 18 (2005) 383-416.

[5] A. Leelarasamee, Recent development in melioidosis, Curr. Opin. Infect. Dis. 17 (2004) 131-136.

[6] S.J. Peacock, Melioidosis, Curr. Opin. Infect. Dis. 19 (2006) 421-428.

[7] W.J. Wiersinga, P.T. van der, N.J. White, N.P. Day, S.J. Peacock, Melioidosis: insights into the pathogenicity of Burkholderia pseudomallei, Nat. Rev. Microbiol. 4 (2006) 272-282.

[8] M.T. Holden, R.W. Titball, S.J. Peacock, A.M. Cerdeno-Tarraga, T. Atkins, L.C Crossman, T. Pitt, C. Churcher, K. Mungall, S.D. Bentley, M. Sebaihia, N.R. Thomson, N. Bason, I.R. Beacham, K. Brooks, K.A. Brown, N.F. Brown, G.L. Challis, I. Cherevach, T. Chillingworth, A. Cronin, B. Crossett, P. Davis, D. DeShazer, T. Feltwell, A. Fraser, Z. Hance, H. Hauser, S. Holroyd, K. Jagels, K.E. Keith, M. Maddison, S. Moule, C. Price, M.A. Quail, E. Rabbinowitsch, K. Rutherford, M. Sanders, M. Simmonds, S. Songsivilai, K. Stevens, S. Tumapa, M. Vesaratchavest, S. Whitehead, C. Yeats, B.G. Barrell, P.C. Oyston, J. Parkhill, Genomic plasticity of the causative agent of melioidosis, Burkholderia pseudomallei, Proc. Natl. Acad. Sci. U. S. A. 101 (2004) 14240-14245.

[9] S. Tumapa, M.T. Holden, M. Vesaratchavest, V. Wuthiekanun, D. Limmathurotsakul, W. Chierakul, E.J. Feil, B.J. Currie, N.P. Day, W.C. Nierman, S.J. Peacock, Burkholderia pseudomallei genome plasticity associated with genomic island variation, BMC Genomics 9 (2008) 190.

[10] K.E. Keith, P.C. Oyston, B. Crossett, N.F. Fairweather, R.W. Titball, T.R. Walsh, K.A Brown, Functional characterization of OXA-57, a class D beta-lactamase from Burkholderia pseudomallei, Antimicrob. Agents Chemother. 49 (2005) 1639-1641.

[11] C. Tribuddharat, R.A. Moore, P. Baker, D.E. Woods, Burkholderia pseudomallei class a beta-lactamase mutations that confer selective resistance against ceftazidime or clavulanic acid inhibition, Antimicrob. Agents Chemother. 47 (2003) 2082-2087.

[12] R.J. Ellis, Chaperomics: in vivo GroEL function defined, Curr. Biol. 15 (2005) R661-R663.

[13] Y.C. Tang, H.C. Chang, A. Roeben, D. Wischnewski, N. Wischnewski, M.J. Kerner, F.U. Hartl, M. Hayer-Hartl, Structural features of the GroEL-GroES nano-cage required for rapid folding of encapsulated protein, Cell 125 (2006) 903-914.

[14] S.E. Radford, GroEL: more than Just a folding cage, Cell 125 (2006) 831-833. 